Quim. Nova, Vol. 34, No. 1, 106-110, 2011

\title{
ELETRODOS POSITIVOS TUBULARES PARA BATERIAS CHUMBO ÁCIDAS PRODUZIDAS COM MATERIAL ATIVO NANOMÉTRICO
}

\author{
Patrício R. Impinnisi*, Juliano de Andrade e Rodrigo V. Palmer \\ Instituto de Pesquisa Para o Desenvolvimento, Centro Politécnico, Universidade Federal do Paraná, CP 19067, 81531-980 Curitiba \\ - Paraná, Brasil
}

Recebido em 13/5/10; aceito em 16/7/10; publicado na web em 16/11/10

\begin{abstract}
LEAD ACID BATTERIES TUBULAR POSITIVE ELECTRODES ASSEMBLED WITH NANOMETRIC ACTIVE MATERIAL. Conventional stationary lead acid batteries positive tubular plates have a specific capacity of about $120 \mathrm{Ah} / \mathrm{kg}$. This value represents an active material utilization coefficient of $50 \%$. The production of these plates includes some initial processes to generate the active $\mathrm{PbO}_{2}$ from a precursor material. In the present work it will be presented a proper and novel methodology to assemble tubular plates directely with nanometric powder of $\mathrm{PbO}_{2}$ particles. The utilization coefficient of these plates was about $80 \%$, and they were able to endure more than 130 severe duty cycles. This high utilization coefficient is a higly desirable feature for electric vehicles batteries.
\end{abstract}

Keyword: lead acid batteries; tubular positive electrode; nanometric $\mathrm{PbO}_{2}$.

\section{INTRODUÇÃO}

O material ativo de placas tubulares convencionais de baterias chumbo ácido se constitui de uma rede interconectada de partículas de $\mathrm{PbO}_{2}$ com diâmetro médio da ordem de microns e área superficial de aproximadamente $2 \mathrm{~m}^{2} / \mathrm{g} .{ }^{1}$ A estrutura de partículas agregadas, que pode ser descrita pelo "modelo de aglomerado de esferas", ${ }^{2}$ é gerada no processo convencional de formação da placa, ou seja, partículas desconexas de um material precursor (uma mistura de $\mathrm{PbO}+\mathrm{Pb}_{3} \mathrm{O}_{4}$ ) são submetidas aos processos de cura e formação para produzir partículas interconectadas de $\mathrm{PbO}_{2}$ ativo. A resistência mecânica e elétrica, assim como o coeficiente de utilização da placa, dependem de uma boa conexão entre as partículas e, portanto, dos processos de cura e formação.

Pavlov e colaboradores ${ }^{3}$ mostraram ser possível montar placas tubulares diretamente a partir do $\mathrm{PbO}_{2}$, desde que as placas tenham uma densidade mínima de $3,8 \mathrm{~g} / \mathrm{cm}^{3}$. Essas placas foram capazes de fornecer até $80 \mathrm{Ah} / \mathrm{kg}$ de $\mathrm{PbO}_{2},{ }^{3}$ sendo que a capacidade específica de placas tubulares convencionais pode chegar a $120 \mathrm{Ah} / \mathrm{kg}$ de $\mathrm{PbO}_{2}{ }^{4}$

Em outro trabalho, Bervas e colaboradores ${ }^{5}$ produziram eletrodos tubulares a partir de $\mathrm{PbO}_{2}$ sintetizado pela oxidação de $\mathrm{Pb}^{2+}$ por persulfato de amônio em meio alcalino. Estes eletrodos forneceram até $100 \mathrm{Ah} / \mathrm{kg}$ de $\mathrm{PbO}_{2}$ por cerca de 100 ciclos, porém, sem ser especificado o regime de descarga utilizado.

No presente trabalho, eletrodos positivos foram produzidos diretamente a partir de $\mathrm{PbO}_{2}$ nanométrico, sem processos eletroquímicos de cura e formação. Na sequência, métodos químicos distintos foram aplicados a estes eletrodos com o objetivo de criar as conexões entre as partículas. Os métodos foram idealizados considerando o modelo de esferas e baseados no mecanismo de dissolução parcial do $\mathrm{PbO}_{2}$ seguida da sua reprecipitação, que deve ocorrer preferencialmente nos contatos entre as partículas, formando os pescoços (os necks previstos pela teoria do aglomerado de esferas). As eficiências destes métodos foram julgadas comparando-se os resultados de medidas de capacidade específica dos diferentes eletrodos. O método mais eficiente, dentre os testados, foi utilizado para produzir eletrodos que posteriormente

*e-mail: rodolfo@lactec.org.br foram testados quanto a sua vida útil (quantidade de ciclos de carga e descarga que o eletrodo suporta antes que a sua capacidade atinja um valor mínimo de $80 \%$ da capacidade estabilizada inicial).

\section{PARTE EXPERIMENTAL}

$\mathrm{O} \mathrm{PbO}_{2}$ nanométrico foi sintetizado pela hidrólise do acetato de chumbo (IV), de acordo com o método desenvolvido por Caballero e colaboradores. ${ }^{6} \mathrm{O}$ produto foi caracterizado por difração de raios $\mathrm{X}$ e o padrão obtido corresponde àquele do $\beta-\mathrm{PbO}_{2}{ }^{7}$ Utilizando a Equação de Scherrer e os parâmetros do pico mais intenso do difratograma pôde-se estimar o tamanho médio das partículas como sendo aproximadamente $10 \mathrm{~nm}^{8}$

A Figura 1 mostra a imagem de MET do material sintetizado, o que confirma a estimativa do tamanho das partículas.

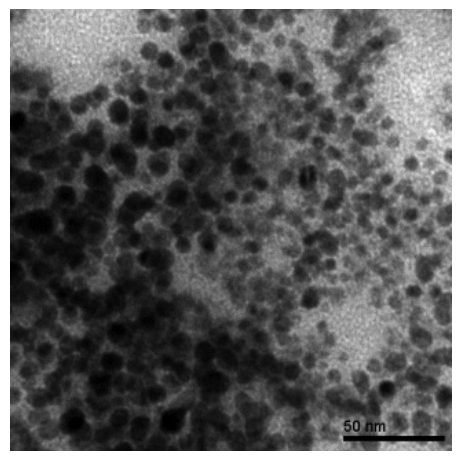

Figura 1. Imagem de MET das partículas de $\mathrm{PbO}_{2}$ recém-sintetizado

A área superficial do material sintetizado foi medida por adsorção de $\mathrm{N}_{2}$ e resultou em $32 \mathrm{~m}^{2} / \mathrm{g}$.

Os eletrodos foram montados da seguinte forma: tubos de contenção que são normalmente utilizados em eletrodos de baterias comerciais foram cortados para se obter um tubo de $25 \mathrm{~mm}$ de altura e 8,6 $\mathrm{mm}$ de diâmetro. A extremidade inferior foi fechada por uma rolha de resina epóxi de cura lenta (cuja resistência à ação do ácido sulfúrico já foi verificada previamente), foi colocada uma haste de chumbo 
(99,9\% de pureza) de $3 \mathrm{~mm}$ de diâmetro e aproximadamente $80 \mathrm{~mm}$ de altura, centralizada em relação ao tubo e fixa na rolha inferior. $\mathrm{O}$ volume entre o tubo e a haste foi preenchido com 2,5 g de $\mathrm{PbO}_{2}$ nanométrico, resultando em uma densidade de aproximadamente 3,5 $\mathrm{g} / \mathrm{cm}^{3}$ e, por fim, a parte superior do eletrodo foi fechada com outra rolha de resina (vazada para a passagem da haste).

Diferentes métodos para criar a estrutura interconectada de partículas foram testados quanto à capacidade específica obtida do material ativo utilizado. Estes métodos estão descritos a seguir:

(a) eletrodo montado com o $\mathrm{PbO}_{2}$ nanométrico, colocado em uma solução de ácido sulfúrico 2,0 mol/L por 1 h e em seguida submetido à medida de capacidade (iniciados com uma descarga, segundo método descrito na sequência);

(b) semelhante ao método anterior, porém, antes da medida de capacidade, o eletrodo foi submetido a um período de polarização anódica galvanostática $\left(\mathrm{i}=10 \mathrm{~A} / \mathrm{Kg}\right.$ de $\mathrm{PbO}_{2}$ ) para a formação de uma camada de corrosão na haste;

(c) eletrodo montado com o $\mathrm{PbO}_{2}$ nanométrico, mantido por $2 \mathrm{~h}$ imerso em água, seco por $30 \mathrm{~h}$ a $75^{\circ} \mathrm{C}$, colocado na célula eletroquímica, submetido a um período de polarização anódica galvanostática para a formação de uma camada de corrosão na haste e submetido ao mesmo procedimento de medida de capacidade dos itens (a) e (b);

(d) eletrodo montado com o $\mathrm{PbO}_{2}$ nanométrico, mantido por $2 \mathrm{~h}$ imerso em solução de $\mathrm{H}_{2} \mathrm{SO}_{4} 2,0 \mathrm{~mol} / \mathrm{L}$, enxaguado por imersão em um béquer com excesso de água deionizada, seco por $30 \mathrm{~h}$ a $75^{\circ} \mathrm{C}$, colocado na célula eletroquímica, submetido a um período de polarização anódica galvanostática para a formação de uma camada de corrosão na haste e submetido ao mesmo procedimento de medida de capacidade que os itens anteriores;

(e) eletrodo submetido a um processo idêntico ao descrito em (d), mas com uma solução do ácido sulfúrico, tanto na imersão prévia quanto na determinação da capacidade, de 4,6 mol/L.

A medida de capacidade específica ao final dos procedimentos descritos anteriormente foi feita submetendo-se a placa a repetidos ciclos de carga e descarga, até que o valor medido de capacidade se repetisse, dentro de uma margem de $4 \%$, por no mínimo três ciclos consecutivos. As densidades de corrente utilizadas foram $\mathrm{i}_{\text {carga }}=10$ $\mathrm{A} / \mathrm{kg}$ de $\mathrm{PbO}_{2}$ e $\mathrm{i}_{\text {descarga }}=5 \mathrm{~A} / \mathrm{kg}$ de $\mathrm{PbO}_{2}$.

Além da medida da capacidade específica, outro método para avaliar o estado de agregação das partículas foi o de retirar o tubo de contenção, voltar a medir a capacidade do eletrodo e comparála com a inicial (quanto menor a perda de capacidade observada melhor o estado de agregação da estrutura gerada pelo procedimento aplicado).

O eletrodo submetido ao método descrito em (d) (que foi o que apresentou o melhor resultado nos ensaios de medida de capacidade, como será mostrado adiante) foi testado quanto à vida útil em dois regimes diferentes de ciclagem: ciclagem superficial (5\% de profundidade de descarga) e, também, de descarga profunda (descarregado até o potencial de corte de $0,8 \mathrm{~V}$ em um regime de descarga de $30 \mathrm{~h}$, $100 \%$ de profundidade de descarga).

Foram utilizadas células eletroquímicas convencionais de três eletrodos. Todos os potenciais foram medidos e serão referenciados ao eletrodo de $\mathrm{Hg} / \mathrm{Hg}_{2} \mathrm{SO}_{4} / \mathrm{H}_{2} \mathrm{SO}_{4}$, na concentração da solução de trabalho (os potenciais dos eletrodos de $\mathrm{Hg} / \mathrm{Hg}_{2} \mathrm{SO}_{4} / \mathrm{H}_{2} \mathrm{SO}_{4} 2,0 \mathrm{~mol} / \mathrm{L}$ e $\mathrm{Hg} / \mathrm{Hg}_{2} \mathrm{SO}_{4} / \mathrm{H}_{2} \mathrm{SO}_{4} 4,6 \mathrm{~mol} / \mathrm{L}$ correspondem a 647 e $586 \mathrm{mV}$ contra o SHE, respectivamente).

Foram utilizados reagentes P. A. marca Merck tanto na síntese do $\mathrm{PbO}_{2}$ quanto na preparação das soluções de ácido sulfúrico. O chumbo utilizado na confecção das hastes foi fornecido pela Tamarana Metais com 99,9\% de pureza. Os experimentos eletroquímicos foram realizados em um equipamento automatizado para testes de baterias fornecido pela Digatron Firing-Circuits, modelo MBT5-05-16V, controlado pelo software BTS 600 .

\section{RESULTADOS E DISCUSSÃO}

\section{Medidas da capacidade específica}

A Figura 2 mostra a evolução da capacidade específica para um eletrodo submetido ao processo descrito em (a) e para um eletrodo submetido ao processo descrito em (b). Como afirmado anteriormente, as densidades de corrente foram $\mathrm{i}_{\text {carga }}=10 \mathrm{~A} / \mathrm{kg}$ de $\mathrm{PbO}_{2}$ e $\mathrm{i}_{\text {descarga }}=$ $5 \mathrm{~A} / \mathrm{kg}$ de $\mathrm{PbO}_{2}$.

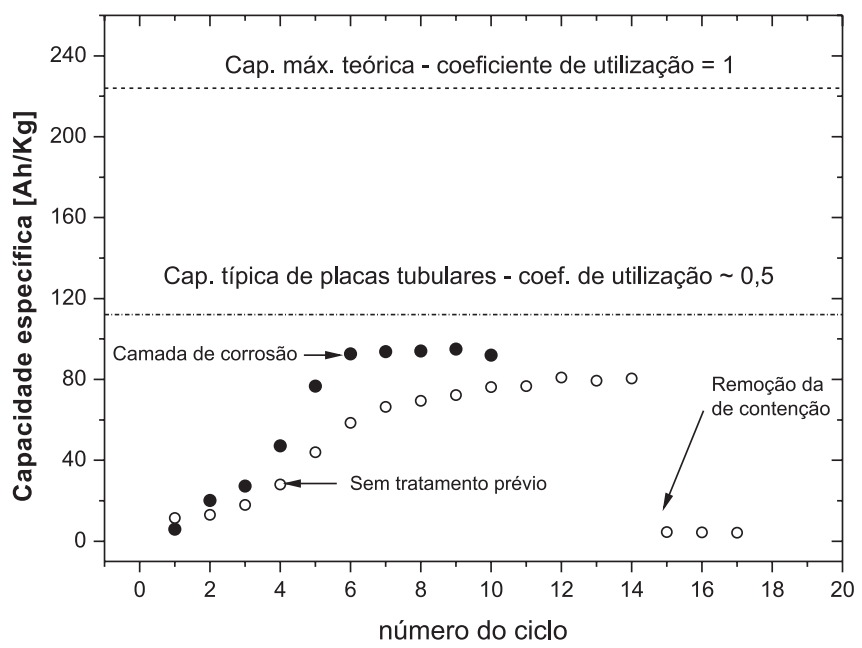

Figura 2. Capacidade específica durante ciclos de carga e descarga para o eletrodo submetido ao tratamento (a) (०) e para o eletrodo submetido ao tratamento $(b)(\bullet)$. No ponto indicado por uma seta o tubo de contenção foi retirado

As linhas horizontais no gráfico mostram os valores de capacidade máxima teórica (224 Ah/kg de $\mathrm{PbO}_{2}$, ou seja, supondo que todo o $\mathrm{PbO}_{2}$ participasse da descarga) e da capacidade típica de eletrodos tubulares (aproximadamente $112 \mathrm{Ah} / \mathrm{kg}$ de $\mathrm{PbO}_{2}$, ou $50 \%$ de utilização do material ativo).

Nesta figura pode-se ver que a capacidade específica do eletrodo que não foi submetido a nenhum tipo de pré-tratamento aumenta nos primeiros ciclos e se estabiliza em um valor inferior àquele de placas tubulares convencionais (como indicado no gráfico pela linha pontilhada), mas em concordância com os resultados apresentados por Pavlov para eletrodos tubulares montados diretamente a partir do $\mathrm{PbO}_{2}{ }^{3}$ Este aumento da capacidade indica que houve a formação de algum contato elétrico entre as partículas, mas ao ser retirado o tubo de contenção o eletrodo se desintegrou perdendo quase completamente sua capacidade, como pode ser visto nesta figura.

$\mathrm{O}$ valor da capacidade do eletrodo submetido ao pré-tratamento (b) se estabilizou mais rapidamente e, de modo geral, apresentou um comportamento semelhante àquele do pré-tratamento (a), com o valor de capacidade aquém do valor tipicamente esperado para placas convencionais. A oxidação da haste realizada no pré-tratamento (b) tem a finalidade de melhorar o contato elétrico entre ela e as partículas de $\mathrm{PbO}_{2}$ adjacentes. Pelos resultados apresentados na Figura 2, este procedimento foi incapaz de garantir um bom contato elétrico entre as partículas do próprio material ativo, como pode ser visto pelo baixo valor do coeficiente de utilização (aproximadamente 36\%).

A seguir serão comparados os diferentes métodos aplicados para agregar as partículas de $\mathrm{PbO}_{2}$. Na Figura 3 são apresentados os resultados das medidas de capacidade específica dos eletrodos submetidos aos pré-tratamentos (c), (d) e (e). 


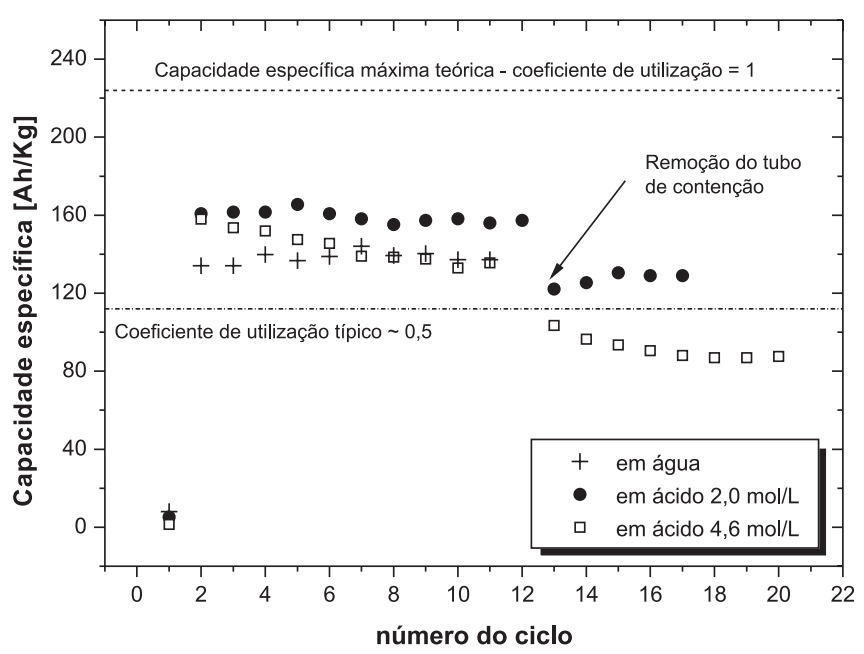

Figura 3. Capacidade específica durante ciclos de carga e descarga para o eletrodo submetido aos pré-tratamentos $(c)(+) ;(d)(\bullet) e(e)(\square)$. No ponto indicado por uma seta o tubo de contenção foi retirado

Em todos os casos é possível verificar que a capacidade específica estabilizada (130-160 Ah/kg de $\mathrm{PbO}_{2}$ ) é maior do que a das placas convencionais (112 Ah/kg de $\mathrm{PbO}_{2}$ ).

A capacidade do eletrodo pré-tratado em água deionizada se estabiliza rapidamente em um valor inferior às capacidades dos demais.

No caso do eletrodo submetido ao pré-tratamento (d), em solução de ácido sulfúrico 2,0 mol/L, é possível verificar que o valor da capacidade específica do eletrodo se estabiliza já nos primeiros ciclos e se mantém alta mesmo após a retirada do tubo de contenção, mostrando que este procedimento foi o mais eficiente na formação das conexões entre as partículas. Neste caso, a perda de material ativo durante o procedimento de retirada do tubo de contenção foi de apenas $7 \%$, indicando um bom estado de agregação das partículas.

O valor da capacidade, do eletrodo submetido ao pré-tratamento (e) em ácido 4,6 mol/L, diminui ao longo dos primeiros ciclos, estabilizando em um valor aproximadamente $17 \%$ inferior ao seu valor inicial. Neste caso, após a remoção do tubo de contenção, a capacidade cai consideravelmente e se estabiliza em um valor de aproximadamente $80 \mathrm{Ah} / \mathrm{kg}$ de $\mathrm{PbO}_{2}$. Cabe salientar que o desprendimento de material ativo durante o processo de remoção do tubo é levado em consideração no cálculo do valor da capacidade específica mostrada na Figura 3.

A capacidade específica inicial obtida nos pré-tratamentos (d) e (e) foi similar (160 Ah/kg de $\mathrm{PbO}_{2}$ ) e superior ao valor típico de placas tubulares convencionais (120 Ah/kg de $\mathrm{PbO}_{2}$ ).

A capacidade específica dos eletrodos submetidos ao prétratamento em ácido mais concentrado $(4,6 \mathrm{~mol} / \mathrm{L}$, procedimento (e)) apresentou uma diminuição considerável ao longo dos primeiros ciclos de carga e descarga e, portanto, uma performance inferior ao procedimento com ácido mais diluído $(2,0 \mathrm{~mol} / \mathrm{L}$, procedimento $(\mathrm{d}))$. O mesmo comportamento foi verificado por Bervas e colaboradores em seu trabalho com eletrodos tubulares, ${ }^{5}$ que sugeriram que a diminuição da capacidade pode estar relacionada com algum processo de sulfatação irreversível, devida à elevada relação $\mathrm{HSO}_{4}-/ \mathrm{PbO}_{2}$.

A causa da elevada capacidade específica medida no presente trabalho, se comparada com os valores convencionais, foi atribuída ao seguinte fato: em uma descarga profunda e lenta, o que limita a descarga e, portanto, a capacidade é a espessura do filme de passivação formado sobre as partículas de $\mathrm{PbO}_{2}{ }^{9}$ Placas distintas, mas descarregadas nas mesmas condições (densidade de corrente real, concentração de ácido, temperatura) terão, ao final da descarga, suas partículas recobertas por filmes de $\mathrm{PbSO}_{4}$ de mesma espessura. Se nestas placas a relação área/ volume das partículas for diferente (diferentes diâmetros), uma maior quantidade de $\mathrm{PbSO}_{4}$ por unidade de massa de material ativo se formará naquela com maior relação área/volume e esta apresentará maior capacidade para uma mesma quantidade de $\mathrm{PbO}_{2}$ inicial.

Após os ciclos mostrados na Figura 3 o eletrodo foi recarregado, desmontado e suas partículas novamente analisadas por microscopia de transmissão com o objetivo de verificar o seu tamanho médio e, também, as suas interconexões. As imagens obtidas podem ser vistas na Figura 4.

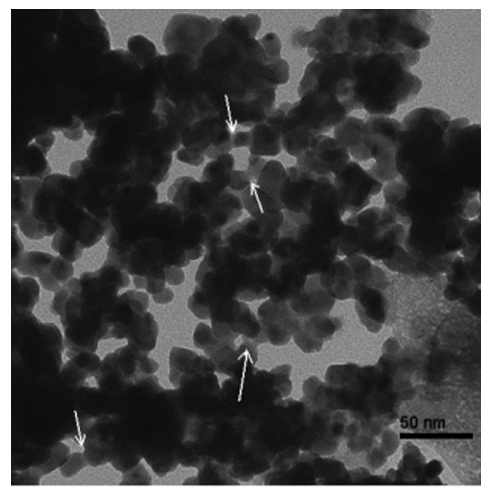

Figura 4. Imagens de microscopia eletrônica de varredura do material ativo após as medidas de capacidade específica mostradas na Figura 3

Como se observa na Figura 4, o tamanho médio das partículas aumenta se comparado com o do material ativo recém-sintetizado (ver Figura 1), mas se mantém na ordem de $20 \mathrm{~nm}$. Nota-se também uma maior quantidade de aglomerados e interconexões (pescoços) indicados por setas brancas na Figura 4.

A área superficial medida do material ativo nesta situação foi de $20 \mathrm{~m}^{2} / \mathrm{g}$.

\section{Avaliação da vida útil}

Sabe-se que, em termos gerais, quanto maior o coeficiente de utilização de um eletrodo de bateria chumbo ácido, menor a sua vida útil. ${ }^{10}$ Por isso, os eletrodos montados com $\mathrm{PbO}_{2}$ nanométrico também foram testados quanto à vida útil frente a dois protocolos de ciclagem diferentes. O primeiro consistindo de uma descarga superficial, de $1 \mathrm{~h}$ de duração (5\% de profundidade de descarga), seguida de recarga (devolvendo uma carga elétrica 30\% superior à retirada na descarga prévia). Após 100 destes ciclos, o eletrodo foi submetido a uma descarga profunda, até o potencial de corte de $0,8 \mathrm{~V}$ em $30 \mathrm{~h}$, para medir a sua capacidade e avaliar a sua degradação.

Um eletrodo montado com $\mathrm{PbO}$ segundo um pré-tratamento convencional, que sabidamente resulta em uma elevada capacidade e elevada vida útil, ${ }^{11}$ também foi colocado para ciclar nas mesmas condições para que se pudesse comparar com o resultado do eletrodo de $\mathrm{PbO}_{2}$ nanométrico.

A Figura 5 mostra os valores das capacidades de ambos os eletrodos medidos a cada 100 ciclos de carga/descarga num regime de ciclagem superficial.

Evidentemente como o eletrodo de $\mathrm{PbO}$ não foi submetido à formação, a sua primeira medida de capacidade apresentou um valor 0 , diferente do eletrodo montado com $\mathrm{PbO}_{2}$ nanométrico que mesmo sem ser submetido a um processo de formação apresentou um valor de capacidade inicial de $20 \mathrm{Ah} / \mathrm{kg}$ de $\mathrm{PbO}_{2}$.

Depois de 600 ciclos ambos os eletrodos apresentam o valor máximo de capacidade (160 Ah/kg para o eletrodo de $\mathrm{PbO}_{2}$ nanométrico e $140 \mathrm{Ah} / \mathrm{kg}$ para o de $\mathrm{PbO}$ ), ambos superiores aos valores típicos apresentados na literatura $(120 \mathrm{Ah} / \mathrm{kg})$. O experimento foi 


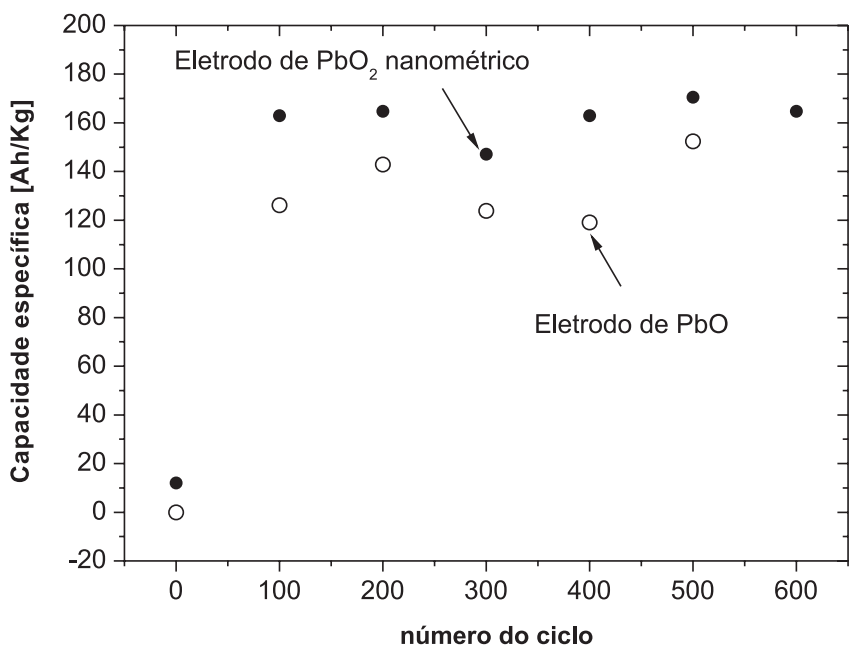

Figura 5. Evolução da capacidade específica ao longo de ciclos de carga e descarga superficial para dois tipos de eletrodos: tubulares montados com $\mathrm{PbO}_{2}$ nanométrico $(\bullet)$ e eletrodos convencionais de $\mathrm{PbO}$ curados (०)

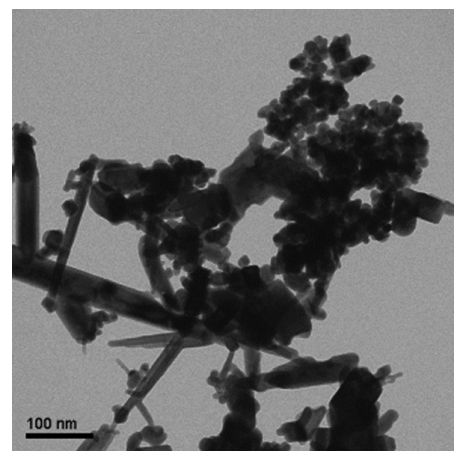

Figura 6. Imagens de microscopia eletrônica de varredura do material ativo do eletrodo de $\mathrm{PbO}_{2}$ nanométrico após as ciclagens mostradas na Figura 5

interrompido após 600 ciclos, apesar de nenhum dos eletrodos ter apresentado problemas, para que se pudesse retirar o material ativo da placa positiva do $\mathrm{PbO}_{2}$ nanométrico e analisar a sua estrutura por microscopia eletrônica de varredura. A imagem do material ativo nestas condições pode ser vista na Figura 6.

Na Figura 6 podem ser vistos alguns cristais em forma de agulhas, que podem ser de sulfato de chumbo não oxidado durante a carga, que se formam com diferentes morfologias, dependendo dos gradientes de concentração no interior da placa. ${ }^{12}$ Podem ser inclusive cristais de sulfatos básicos de chumbo, cuja formação é possível devido ao aumento de $\mathrm{pH}$ no interior dos poros do material ativo durante a descarga. ${ }^{13}$ Estes cristais podem ser ainda $\mathrm{PbO}_{2}$ que tenha se formado a partir dos sulfatos básicos por uma transformação metassomática. Nesta figura também são vistos também vários cristais globulares de $\mathrm{PbO}_{2}$ de menor tamanho.

Ainda na Figura 6 é possível verificar a presença de cristais de $\mathrm{PbO}_{2}$ que vão desde 10 até $150 \mathrm{~nm}$, indicando um aumento no diâmetro dos cristais. É interessante notar também que, mesmo após 600 ciclos de carga e descarga, o tamanho médio dos cristais ainda é muito menor que o de cristais de placas convencionais.

O segundo método utilizado para medir a vida útil das placas tubulares foi concebido para emular uma utilização severa de uma bateria chumbo ácida. O procedimento consistiu em ciclos contendo uma descarga profunda, recarga parcial, nova descarga profunda e finalmente uma carga completa, repetindo os seguintes passos: descarga profunda até o potencial de corte em regime de $30 \mathrm{~h}$; recarga rápida parcial até
$50 \%$ do estado de carga em $2 \mathrm{~h}$; nova descarga profunda até o potencial de corte em regime de $30 \mathrm{~h}$ e, finalmente, recarga completa em $20 \mathrm{~h}$. Uma explicação adicional deve ser fornecida sobre a recarga rápida parcial aqui utilizada: durante a recarga rápida é fornecida à placa uma carga igual a sua capacidade, ou seja, se a capacidade da placa medida numa descarga lenta foi de $280 \mathrm{Ah}$, na recarga rápida devolve-se a esta placa $280 \mathrm{Ah}$ em $2 \mathrm{~h}$. Como a eficiência da recarga rápida é muito baixa, apenas uma fração da carga fornecida é efetivamente assimilada pela placa (aproximadamente 50\%) e, por isso, se referiu a esta carga como "parcial". O restante da carga elétrica fornecida é consumido em reações paralelas de evolução de $\mathrm{O}_{2}$ e oxidação da haste de chumbo.

A Figura 7 mostra a capacidade da placa medida após as recargas completas.

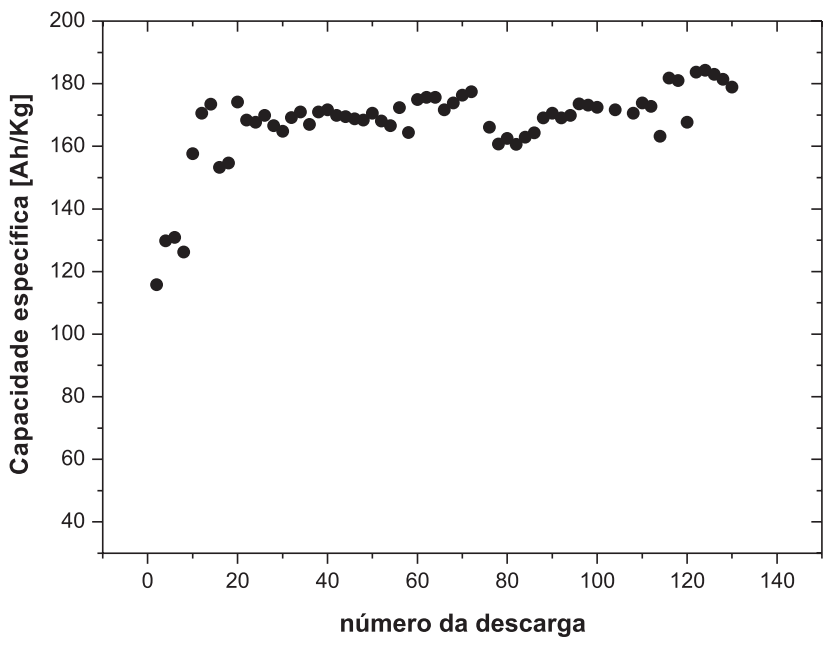

Figura 7. Capacidade específica medida ao longo dos ciclos do protocolo severo de teste de vida útil descrito acima. Os valores mostrados são da capacidade medida após a recarga completa

Apesar dos parâmetros escolhidos para os ciclos (descarga profunda, recarga parcial e elevada quantidade de reações paralelas) favorecerem o envelhecimento prematuro da placa, a capacidade da mesma manteve seu valor máximo durante todo o teste.

Outro fato que chama a atenção é o aumento no valor da capacidade específica, que chegou até a $180 \mathrm{Ah} / \mathrm{kg}$ de $\mathrm{PbO}_{2}$, que implica em um coeficiente de utilização de mais de $80 \%$ do material ativo positivo.

Este aumento foi inicialmente atribuído ao fato de que a recarga rápida é realizada com elevadas correntes, o que implica em submeter o eletrodo positivo a elevados potenciais anódicos. Nesta situação se favorece a corrosão do chumbo da grade e, possivelmente, a diminuição da resistência da interface haste/ material ativo. Como mostrado por Pavlov, ${ }^{10}$ a elevada relação área do material ativo/área da haste implica que esta interface é um gargalo para a corrente elétrica, o que atribui a esta região um peso maior na composição da resistência global da placa. A maior corrosão da grade nos potenciais atingidos durante a carga rápida provavelmente melhorou a condutividade na interface haste/ partículas, criando novos contatos e diminuindo a densidade de corrente nesta região.

Uma questão que ainda poderia ser levantada é a respeito da participação do produto de corrosão da haste de chumbo na elevada capacidade medida (180 Ah/kg). Em um experimento paralelo, uma haste de chumbo, idêntica às usadas na montagem dos eletrodos, foi submetida ao mesmo protocolo de teste severo utilizado e verificouse que a "capacidade" do produto de corrosão da haste foi de no máximo $3 \mathrm{mAh}$, aproximadamente $1 \%$ da capacidade apresentada pelas placas, portanto, foi considerada desprezível. 


\section{CONCLUSÕES}

Mostrou-se que é possível montar placas tubulares positivas de baterias chumbo ácido diretamente a partir de $\mathrm{PbO}_{2}$ nanométrico, aplicando-se um método adequado para criar as conexões entre as partículas.

As placas submetidas ao pré-tratamento desenvolvido neste trabalho e aos ciclos iniciais de carga e descarga apresentaram elevada resistência mecânica de tal forma que, mesmo após se retirar a contenção tubular, as placas mantiveram valores elevados de capacidade durante a ciclagem.

O método desenvolvido para agregar as partículas não aumentou significativamente o tamanho das mesmas, mantendo a elevada relação área/volume do material ativo.

As placas tubulares de $\mathrm{PbO}_{2}$ nanométrico apresentaram vida útil comparável às das convencionais, frente a ciclos de cargas e descargas superficiais.

As placas tubulares de $\mathrm{PbO}_{2}$ nanométrico submetidas a um regime de ciclagem severa mantiveram sua capacidade em valores superiores aos das convencionais.

Atingiram-se valores de até $190 \mathrm{Ah} / \mathrm{kg}$, correspondendo a um coeficiente de utilização do material ativo da ordem de $85 \%$. Com este tipo de tecnologia poderiam ser montadas baterias com a mesma capacidade que as convencionais, mas com menor quantidade de material ativo, o que diminuirá o peso global da bateria, aumentado a sua densidade energética.

\section{AGRADECIMENTOS}

À Companhia Paranaense de Energia, COPEL, pelo suporte financeiro. Ao Instituto de Pesquisa para o Desenvolvimento, LACTEC, pela infraestrutura laboratorial. À Tamarana Metais Ltda pelo fornecimento de insumos. À Sra. R. B. Freitas do Centro de Microscopia da UFPR, pelas análises de MET. E ao MSc. O. Baron, do LACTEC, pelas análises de BET.

\section{REFERÊNCIAS}

1. Pavlov, D.; Bashtavelova, E.; J. Electrochem. Soc. 1986, 133, 241.

2. Winsel, A.; Voss, E.; Hullmeine, U.; J. Power Sources 1990, 30, 209.

3. Pavlov, D.; Dakhouche, A.; Rogachev, T.; J. Power Sources 1993, 42, 71.

4. Pavlov, D.; J. Power Sources 1991, 33, 221.

5. Bervas, M.; Perrin, M.; Geniès, S.; Mattera, F.; J. Power Sources 2007, 173, 570 .

6. Caballero, A.; Cruz, M.; Hernán, L.; Morales, J.; Sánchez, L.; J. Power Sources 2003, 113, 376.

7. Powder diffraction file JCPDS 35-1422.

8. Cullity, B. D.; Elements of X-Ray Diffraction, $1^{\text {st }}$ ed., Addison Wesley: Massachusets, 1956.

9. D’Alkaine, C. V.; Carubelli, A.; Fava, H. W.; Sanhueza, A. C.; J. Power Sources 1995, 53, 289.

10. Pavlov, D.; J. Power Sources 1995, 53, 9.

11. Impinnisi, P. R.; D’Alkaine, C. V.; Andrade, J.; J. Power Sources 2000 , 85, 131.

12. Pavlov, D.; Kirchev, A.; Stoycheva, M.; Monahov, B.; J. Power Sources 2004, 137, 288.

13. Pavlov, D.; Electrochim Acta 1968, 13, 2051. 